\title{
Frictionless indentation of dissimilar elastic-plastic spheres
}

\author{
Sinisa Dj Mesarovic ${ }^{a}$, Norman A Fleck ${ }^{\mathrm{b}, *}$ \\ ${ }^{a}$ Department of Material Science and Engineering, University of Virginia, Charlottesville VA 22903, USA \\ b Department of Engineering, Cambridge University, Trumpington Street, Cambridge CB2 1PZ, UK
}

Received 30 May 1999

\begin{abstract}
A finite element study is performed on the frictionless normal contact of elastic-plastic spheres and rigid spheres. The effects of elasticity, strain hardening rate, relative size of the spheres and their relative yield strength are explored. Indentation maps are constructed, taking as axes the contact size and yield strain, for a wide range of geometries. These show the competing regimes of deformation mechanism: elastic, elastic-plastic, fully plastic similarity and finite deformation regime. The boundaries of the regimes depend upon the degree of strain hardening, relative size of the bodies in contact and upon their relative yield strengths. The regime of practical importance is the finite deformation regime for practical applications such as powder compaction. The contact force-displacement law, to be used as a part of the micromechanical constitutive model for powder compaction, is constructed semi-empirically by scaling the similarity contact law by a factor which depends on the relative size, relative yield strength and the strain hardening exponent of the bodies in contact. The accuracy of the assumption of independent contacts is addressed for the isostatic compaction of an assembly of rigid and deformable spheres, arranged in a B2 unit cell, based on two overlapping simple cubic lattices. Provided that the relative density of the compact is lower than about 0.82 , the contacts deform independently. () 2000 Elsevier Science Ltd. All rights reserved.
\end{abstract}

Keywords: Contact mechanics; Finite element method; Indentation; Plasticity; Power compaction

\section{Introduction}

The elastic-plastic contact of two non-conforming bodies is a fundamental problem in the mechanics of materials, with a wide range of applications. For example, the cold pressing of metallic powders into near net shape parts is by the plastic indentation of deformable particles, and predictions of the multi-axial compaction behaviour are based on the knowledge of the local indentation response between particles. Recently, there has been interest in the development of powder composites, such as silicon carbide particles mixed with aluminium alloy powder, whereby the hard ceramic particles strengthen the soft metallic phase. There is a need to develop accurate constitutive models of compaction in order to optimise tooling design and to eliminate manufacturing defects such as cracking, from regions of tensile residual stress within the part. The purpose of this article is to determine the contact law between powder particles, for subsequent use within compaction models for the powder aggregate. In the early stages of compaction ("Stage I

\footnotetext{
${ }^{*}$ Corresponding author. Tel.: +44-01223-332650; fax: +44-01223-332662.

E-mail address: nafl@eng.cam.ac.uk (N.A. Fleck).
} 
compaction"), the aggregate can be viewed as an assembly of particles, and the macroscopic response can be deduced by suitable homogenisation of the local indentation response between two representative particles. (See for example, Fleck et al. (1992) for rate-independent monolithic powder, and Storakers et al. (1999) for the general viscoplastic composite case.) The assumption that the contacts between particles behave independently plays an essential role in these models. Provided the initial relative density of the aggregate exceeds about 0.3 , the number of contacts between particles is sufficiently high to prevent particle rolling so that the particles undergo a combination of mutual normal indentation and sliding. Fleck et al. (1992) has shown that the shear tractions associated with inter-particle sliding have a negligible effect on the macroscopic response. Thus, a knowledge of the frictionless normal indentation law suffices to determine the compaction response of the aggregate.

A major motivation for the current study is the compaction of a mixture of metallic and ceramic powders. The compaction of metal-ceramic composite powders is complicated by the occurrence of various configurations of ceramic-ceramic, metal-metal and metal-ceramic contacts. We can idealise ceramicceramic contacts and metal-metal contacts by the indentation of two elastic spheres and two elastic-plastic spheres, respectively. For the case of a metal-ceramic contact, we have performed preliminary finite element calculations of mutual indentation of a metallic sphere, with a realistic elastic-plastic constitutive law, and a ceramic sphere with a realistic elastic modulus. We find that the elastic compliance of the ceramic gives a negligible contribution to the indentation response, and we conclude that the ceramic particles can be treated as rigid. Thus, a metal-ceramic contact can be idealised by the indentation of an elastic-plastic sphere by a rigid sphere.

Storakers et al. (1999) have recently developed a model to predict the macroscopic compaction response of a powder composite. The model makes use of the Storakers et al. (1997) similarity solution for the contact of two spheres of differing diameter and made of different viscoplastic solids. This contact law is summarised in Section 1.1 for the case of rate-independent power-law hardening solids. In the present article, we seek to determine the regime of validity for this assumed contact law by modelling directly the mutual indentation of two spheres of unequal diameter and unequal material properties. We find that elasticity, finite deformation, particle shape and the interaction between contacts each can have a significant effect upon the contact law, depending upon the operative regime of indentation.

The present numerical study of inter-particle contact has the following main goals:

- To explore the domain of validity of the similarity solution for the indentation of a rigid sphere against a deformable sphere and for the mutual indentation of two deformable spheres. Specific attention is paid to the effects of elasticity, finite deformation and the relative size of the spheres.

- To establish the limits of validity of the assumption of independent contacts.

- To establish an approximate contact law between two dissimilar particles for the practical range of material parameters, relative particle diameters and the degree of indentation associated with Stage I powder compaction.

In this effort, we are aided by the previous works of Gampala et al. (1994) and Ogbonna and Fleck (1995). Gampala et al. modelled the indentation of a deformable sphere and a rigid half-space. They identified the regimes of indentation, but did not identify the criterion for the onset of finite deformation regime. Ogbonna and Fleck analysed the contact between identical spheres, and constructed the yield surfaces for the compaction of a periodic array of spheres. Here, we perform a systematic study of the effect of relative size and relative strength of particles in contact, and also perform a full three-dimensional analysis of contact interactions for a periodic unit cell.

\subsection{The similarity solution}

Storakers et al. (1997) and Storakers (1997) have developed a similarity solution for the normal indentation of two viscoplastic spheres by extending the Hill et al. (1989) solution for the indentation of a 


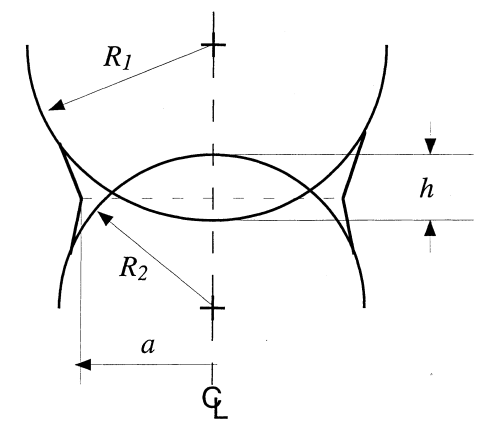

Fig. 1. Geometry of contact between two spherical particles with radii $R_{1}$ and $R_{2}$. A contact radius $a$ is generated for a total overlap $h$.

half-space by a rigid sphere. Here, we summarise the rate-independent version of their similarity solution. The configuration studied by Storakers et al. (1997) is shown in Fig. 1. Sphere 1 of radius $R_{1}$ and sphere 2 of radius $R_{2}$ are pressed together in a frictionless normal indentation, so that the contact radius is $a$ at a total overlap of $h$.

The following simplifying assumptions are introduced to obtain a self-similar solution:

1. The two spheres are composed of rigid-plastic, power-law-solids in accordance with $\mathbf{J}_{2}$ flow theory. In uniaxial tension, the stress $\sigma$ is related to the strain $\varepsilon$ according to

$$
\sigma=\sigma_{i} \varepsilon^{1 / m}, \quad i=1,2,
$$

where sphere 1 has a reference strength $\sigma_{1}$ and sphere 2 has a reference strength $\sigma_{2}$. Both solids have the same strain hardening exponent $(1 \leqslant m \leqslant \infty)$.

2. The contact radius is assumed to be sufficiently small compared to the radius of each sphere that each sphere can be treated as a semi-infinite half-space.

3. Strains and deformations are small, and the spherical profile of the bodies in contact is approximated by a paraboloid of revolution. Then, if the normal displacement of sphere 1 within the contact patch is $u_{1}$ at a radius $r$, and the corresponding normal displacement of sphere 2 is $u_{2}$, conformity of the two surfaces within the contact dictates that

$$
u_{1}+u_{2}=h-\frac{r^{2}}{2 R_{1}}-\frac{r^{2}}{2 R_{2}}
$$

with $h \ll R_{1}$ and $h \ll R_{2}$.

With these restrictions, the indentation solution has the property of self-similarity, i.e., the geometry, stress and strain fields at any stage of indentation can be expressed in terms of an invariant solution. Moreover, the solution to the problem of contact between spherical bodies is a generalisation of the solution for the contact between a rigid sphere and a semi-infinite solid, and is obtained from the latter by appropriate scaling. The method can be generalised to include rate-dependent solids with a response describable by a power law-creep law. The solution for the indentation of a semi-infinite solid by a rigid sphere is provided by Hill (1992), Bower et al. (1993) and Storakers et al. (1997) for a power-law creeping solid, and by Biwa and Storakers (1995) for a $\mathbf{J}_{2}$ flow theory solid.

The scaling law, relating the indentation of the spheres to the indentation of a half-space by a rigid ball, generalises the one used in elastic Hertzian contact. An equivalent radius $R_{0}$ suffices to describe a given geometry, and an equivalent strength $\sigma_{\mathrm{e}}$ describes the combined strengths of the two spheres,

$$
1 / R_{0} \equiv 1 / R_{1}+1 / R_{2}, \quad \sigma_{\mathrm{e}}^{-m} \equiv \sigma_{1}^{-m}+\sigma_{2}^{-m} .
$$


The average pressure is related to the contact radius $a$ by the power-law relation

$$
\frac{F}{\pi a^{2} \sigma_{\mathrm{e}}}=k(m)\left(\frac{a}{R_{0}}\right)^{1 / m},
$$

and the contact area is proportional to the indentation depth,

$$
\frac{h}{R_{0}}=\frac{1}{2 c^{2}(m)}\left(\frac{a}{R_{0}}\right)^{2}
$$

where the constants $c^{2}(m)$ and $k(m)$ depend on $m$, but are independent of the indentation depth, and of the diameters and strengths of the bodies in contact. Biwa and Storakers (1995) tabulated $c^{2}(m)$ and $k(m)$. Their finite element formulation is based on the assumption of self-similarity and are essentially single-step solutions, where the history dependence is replaced by a spatial (radial) dependence.

Relations (1.4) and (1.5) imply that the indentation force depends upon the indentation depth $h$ according to

$$
\frac{F}{\pi R_{0}^{2} \sigma_{\mathrm{e}}}=K(m)\left(\frac{h}{R_{0}}\right)^{1+(1 / 2 m)}
$$

where

$$
K=2^{(1+(1 / 2 m))} c^{(2+(1 / m))} k .
$$

In the present article, we use the spherical, rather than the parabolic shape of the bodies in contact. The differences in the profiles of a sphere and a paraboloid with the same curvature at the apex become significant only for large contacts $a / R>0.4$. Changes in the indentation regimes which we observe occur at smaller contacts $(0.1<a / R<0.3)$, so that our results are not affected by the difference between the spherical and the parabolic shape.

Stress and strain distributions in Storakers et al. (1997) self-similar solution for the contact between the power-law, incompressible, rigid-plastic spheres are, apart from scaling (1.3), identical to the solution for the indentation of a half-space. This can be contrasted with the Sternberg and Rosenthal (1952) singular solution for anti-diametric concentrated load on a linear elastic compressible sphere. Whereas in the limit of infinite radius, the Sternberg and Rosenthal solution does reduce to the Boussinesq solution for half-space, the stress distributions for finite radii have a different singularity from the Boussinesq distribution.

\subsection{Programme of study}

We consider the frictionless normal indentation of dissimilar elastic-plastic spheres. The relative diameter and strength of the two spheres is varied over the full range, and the effects of yield strain and strain hardening exponent are explored. The axisymmetric and three-dimensional configurations studied are shown in Fig. 2. We consider a number of special cases:

(a) indentation of two identical deformable spheres,

(b) indentation of a deformable sphere and a rigid sphere of equal diameter,

(c) a rigid sphere indenting a deformable half-space (Brinell indentation),

(d) a sphere compressed between two flat rigid platens, and

(e) a deformable sphere compressed between two deformable half-spaces.

The infinitely long chain of identical spheres (Fig. 2(a)) has symmetry planes both through the contacts and through the centres of the particles, whereas the alternating configuration of deformable and rigid particles (Fig. 2(b)) has symmetry planes only through the centres of the particles. The configurations shown in Fig. 2(a) and (b) are studied with and without the fixed lateral constraint, i.e. the equatorial radius 


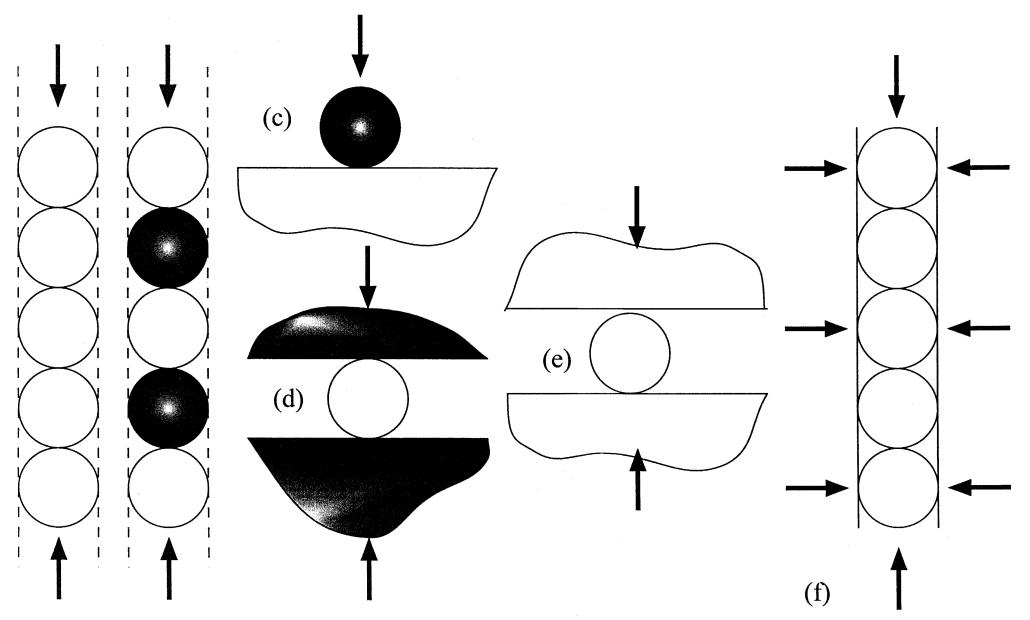

(a) (b)

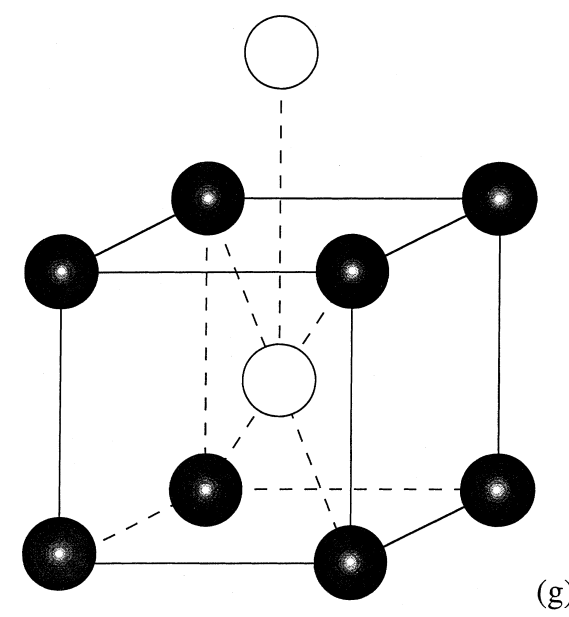

Fig. 2. Axisymmetric configurations: (a) a line of deformable spheres with or without fixed lateral constraint, (b) a line of alternating deformable and rigid spheres with or without fixed lateral constraint, (c) Brinell indentation, (d) a deformable sphere compressed between rigid, frictionless platens, (e) a deformable sphere compressed between deformable half-spaces, (f) a line of deformable spheres in a shrinking tube. Three-dimensional configuration: (g) B2 lattice, initial density $D_{0}=0.68$. Particles are shown smaller than their real size for better visibility. The central deformable sphere has eight initial contacts with rigid spheres.

is either fixed or free to expand. We find that the degree of lateral constraint (illustrated by the dashed lines in Fig. 2(a) and (b)) as practically no effect on the contact behaviour for the range of contact sizes studied. The effect of interaction of contacts is studied by two idealisations of isostatic compaction. First, an axisymmetric array of identical particles is placed within an imaginary shrinking tube (Fig. 2(f)), and a radial strain rate equal to the axial strain rate is imposed. Second, a three-dimensional simple cubic arrangement of deformable and rigid spheres is subjected to isostatic compaction, as shown in Fig. $2(\mathrm{~g})$.

Besides its practical significance in Brinell and Rockwell testing, and its theoretical significance as the most frequently studied case, the configuration in Fig. 2(c) is also the limiting geometry for a small ceramic particle in contact with a larger metallic particle. The opposite limit of contact between a large rigid particle 
and a small deformable particle (Fig. 2(d)) is an identical boundary value problem to that of mutual indentation of equi-sized deformable particles (see Fig. 2(a)). Finally, the configuration in Fig. 2(e) is the limiting case of contact between a very small and a very large deformable particle.

The Brinell indentation problem of Fig. 2(c) has been studied in detail by Mesarovic and Fleck (1999) (referred to as (I) hereafter), and it is shown therein that several stages of indentation exist. Initially, elastic indentation occurs and the classical solution of Hertz is reproduced. The initial yield occurs when the average contact pressure (over the projected contact area) is approximately $10 \%$ above the yield strength of the material. Continuing indentation is characterised as elastic-plastic as both the elastic and plastic deformation contribute to the overall response. Further indentation leads to the fully plastic regime which can be subdivided into two regimes: for relatively small contact sizes, the similarity solution applies, whereas for large contact sizes, a finite deformation mode dominates. In the Brinell problem, the similarity regime is not entered for realistic values of yield strain. It is the purpose of the present article to extend the previous study (I) to the indentation of dissimilar spheres and, in particular, to explore the influence of the relative diameter of the spheres on the regimes of dominance of the competing modes of indentation. We shall show that the contact between spheres fits into the same basic framework as reported in (I).

\section{Constitutive law, finite element implementation and dimensionless groups}

\subsection{Constitutive law}

It is assumed that the deformable solid satisfies $\mathbf{J}_{2}$ flow theory with isotropic hardening, and with the total strain obeying a piecewise linear/power law. In uniaxial tension, the stress $\sigma$ is related to the strain $\varepsilon$ by

$$
\varepsilon=\left\{\begin{array}{ll}
\sigma / E_{i} & \text { for } \sigma \leqslant \sigma_{0 i} \\
\left(\sigma / \sigma_{i}\right)^{m} & \text { for } \sigma>\sigma_{0 i}
\end{array}\right\}, \quad i=1,2
$$

where $\sigma_{i}$ is a representative strength and $E_{i}$ is Young's modulus of the solid $i$; the initial yield stress, $\sigma_{0 i}$ and yield strain, $\varepsilon_{0 i}$ of the solid $i$ are given by

$$
\sigma_{0 i}=\left(\frac{\sigma_{i}^{m}}{E_{i}}\right)^{1 /(m-1)} \quad \text { and } \quad \varepsilon_{0 i}=\sigma_{0 i} / E_{i}=\left(\frac{\sigma_{i}}{E_{i}}\right)^{m /(m-1)} .
$$

At large strains, the plastic deformation is almost identical to the pure power law (1.1):

$$
\varepsilon^{\mathrm{pl}}=\left(\sigma / \sigma_{i}\right)^{m}-\sigma / E_{i}
$$

The constitutive law is chosen so that Young's modulus, $E_{i}$ can be varied without changing the total strain-stress curve at large strains. This is achieved by keeping $\sigma_{i}$ and $m$ constant in Eq. (2.1c). In (I), we compared the indentation of a half-space described by the above constitutive model and the indentation of the half-space described by a Ramberg-Osgood type constitutive model. Differences were observed only in the early stages, i.e. the elastic-plastic regime, where the Ramberg-Osgood case exhibited a more gradual transition to the fully plastic similarity regime. The present model is computationally more convenient and provides a clear demarcation between the elastic and plastic deformation. 
The uniaxial constitutive relations $(2.1 \mathrm{a}-\mathrm{c})$ are readily generalised to the arbitrary stress-strain states within the framework of $\mathbf{J}_{2}$ flow theory by defining the equivalent (Mises) stress $\bar{\sigma}$, the equivalent strain rate $\dot{\bar{\varepsilon}}$, and the equivalent strain $\bar{\varepsilon}$ :

$$
\bar{\sigma}=\sqrt{\frac{3}{2} s_{i j} s_{i j}}, \quad \dot{\bar{\varepsilon}}=\sqrt{\frac{2}{3} D_{i j} D_{i j}}, \quad \bar{\varepsilon}(t)=\int_{0}^{t} \dot{\bar{\varepsilon}}(\tau) \mathrm{d} \tau,
$$

where $s_{i j}$ are the deviatoric components of the Cauchy stress, $D_{i j}$ are the components of the symmetric part of the velocity gradient (with respect to the current configuration), and the usual indicial notation is used.

A series of finite element computations have been performed for the cases shown in Fig. 2. The strain hardening parameter $m$ in Eq. $(2.1 \mathrm{a}-\mathrm{c})$ is assumed to take values 3,5 and $\infty$, with the limiting case $m=\infty$ representing an elastic-ideally plastic solid. The magnitude of the indentation depth $h$ is chosen to cover the range of stage I powder compaction, wherein the aggregate can be treated as discrete particles with identifiable contacts. Assuming affine deformation and isostatic compaction of particles of equal radius $R$, the relative density, $D=$ (solid volume/total volume), is related to the inter-penetration $h$ by

$$
D=D_{0}\left(1-\frac{h}{2 R}\right)^{-3}
$$

where $D_{0}$ designates the initial relative density. For $D_{0}=0.64$, which corresponds to the dense random packing of identical spheres, the maximum value of indentation depth $h=0.2 R$ used here, corresponds to $D=0.88$. Typically, stage I compaction ends at a relative density $D \approx 0.8$, as discussed by Fleck et al. (1992).

\subsection{Finite element implementation}

The finite element calculations were performed using the commercial finite element code ABAQUS (1995). Details of the formulation can be found in the ABAQUs Theory Manual, (1995). Here, we only mention that the finite deformation and rotations are treated with an "updated Lagrangian" formulation in that the configuration at the beginning of each increment is the reference configuration and that the objective (Jaumman) stress rate measure is used to account for the material rotation.

The contact surface option and the rigid surface option were used, and a typical axisymmetric mesh for the deformable particle is shown in Fig. 3(a). The number of load steps varied between 150 and 1000. In order to maximise the accuracy, the nodal spacing along the contact surface starts at $0.0015 R$ and gradually increases along the perimeter. To verify the results at small contact sizes, a mesh of double density was used for selected cases. The mesh for the case of Brinell indentation has already been discussed in (I), whereas the mesh for a deformable sphere in contact with a deformable half-space (Fig. 2(e)) is a combination of the meshes shown in Fig. 3(a) and in (I). The three-dimensional mesh for the B2 unit cell is shown in Fig. 3(b). It has a shortest distance between the nodes of $0.005 R$ at the location of initial contact. A single run with $m=3$ was performed and it required 145 increments.

The error associated with the discrete increment in contact size is discussed in detail in (I). Here, we summarise only the main points. As the indentation depth $h$ increases, successive surface nodes come into contact and the contact size increases in discrete steps. Thus, in the loading interval over which the number of contacting nodes is constant, the indentation depth $h$ increases but the contact radius $a$ remains almost constant. On a plot with contact radius $a$ as abscissa and $h$ as ordinate, the data points appear as vertical columns, with each column corresponding to a constant number of nodes in contact. The height of the columns (scatter bands) scales with the ratio of node spacing along the contact surface and the contact radius, $a$. In (I), we compared the finite element results with the elastic Hertzian solution for the average 
(a)

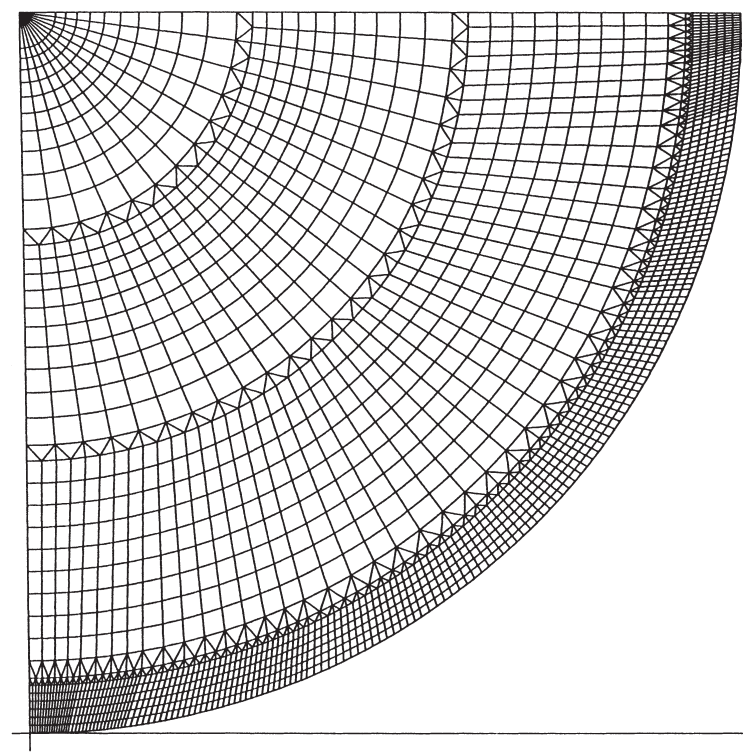

(b)

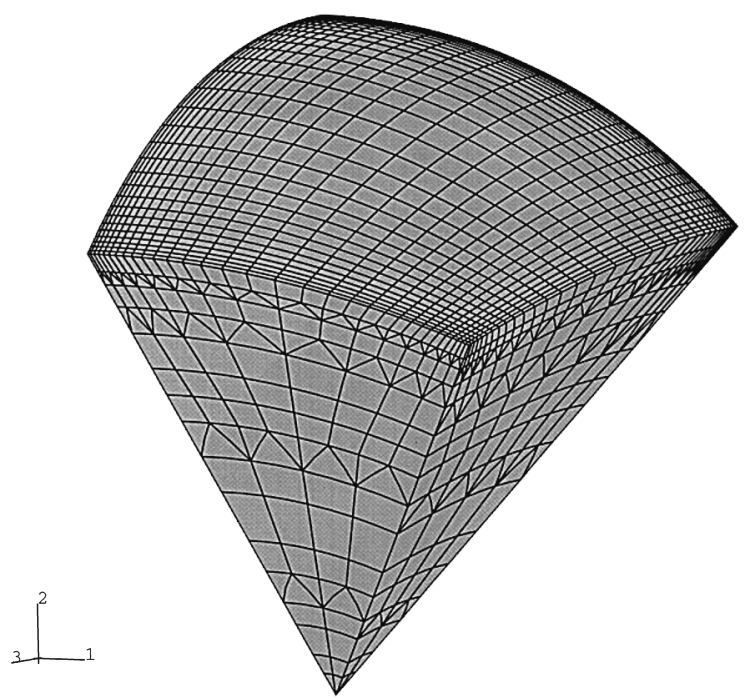

Fig. 3. Finite element meshes: (a) axisymmetric mesh with about 2500 second order elements and 23,500 degrees of freedom; eight-node rectangles and six-node triangles for transition. The shortest distance between the nodes at initial contact is $1.5 \times 10^{-3} R$. (b) threedimensional mesh for isostatic compaction of the B2 lattice of soft and hard particles shown in Fig. 1 (f). 1/24-th of the soft sphere is meshed. The mesh comprises about 4300 second order elements and 100,000 degrees of freedom; 20-node bricks and 15-node triangular prisms for transition. Mid-face nodes are added to the brick elements on the contact surface. The shortest distance between the nodes at initial contact is $5 \times 10^{-3} R$.

pressure and $\left(a^{2} / 2 h R_{0}\right)$, and concluded that the mid-points in the bands are the most accurate. For clarity, only the mid-points are reported here. For the mesh in Fig. 3(a), the scatter in $h$ is $\pm 7.5 \%$ at $a / R=0.01$. The mesh was designed so that the scatter quickly decreases to under $\pm 2.5 \%$ at $a / R>0.08$. Similar col- 
umns of data points will appear in the plots of force, average pressure and $\left(a^{2} / 2 h R_{0}\right)$ against the contact radius, $a$.

\subsection{Presentation of results in terms of dimensionless groups}

The appropriate choice of non-dimensional groups to characterise the competing regimes of deformation requires consideration. For the case of Brinell indentation of an elastic-plastic half-space by a rigid indenter, it was shown in (I) that, with increasing indent depth, the dominant deformation mechanism evolves from the elastic Hertzian regime, through elastic-plastic and fully plastic similarity regimes, to the finite deformation regime. Within the elastic regime, the indentation pressure scales with $\left(E^{*} a / R\right)$, where $a$ is the contact radius, $R$ is the radius of the ball indenter; $E^{*}$ is the plane strain modulus and is related to Young's modulus $E$ and to Poisson's ratio $v$ by $E^{*} \equiv E /\left(1-v^{2}\right)$. The transition between the elastic regime and the elastic-plastic regime, and the transition between the elastic-plastic regime and the similarity regime occur when $a E^{*} / R \sigma_{0}$ attains critical values, where $\sigma_{0}$ is the initial yield strength of the half-space. The transition between the similarity regime and the finite deformation regime is characterised by a critical value of the normalised contact radius $a / R$.

The question arises whether a more general scaling exists for the geometries of Fig. 2. We shall adopt the combined elastic modulus for the two contacting bodies which is rigorously valid in the Hertzian limit,

$$
\frac{1}{E^{*}}=\frac{1-v_{1}^{2}}{E_{1}}+\frac{1-v_{2}^{2}}{E_{2}},
$$

where $E_{1}, E_{2}, v_{1}$ and $v_{2}$ are Young's moduli and Poisson ratios of the two bodies. The combined radius of curvature is taken as $R_{0}$, as defined in Eq. (1.3), and as suggested by both the Hertz solution and by the similarity solution. Further, with $R_{0}$ defined by Eq. (1.3), yield initiates when $a E^{*} / R_{0} \sigma_{0}$ reaches a critical value. Here, $\sigma_{0}$ is the lower of the two initial yield strengths. We shall show that the behaviour in the elasticplastic regime is governed by the magnitude of $a E^{*} / R_{0} \sigma_{0}$ for all the cases shown in Fig. 2.

By analogy with Brinell indentation (I), it might be thought that the onset of the finite deformation regime is given by a critical value of $a / R_{0}$. However, a careful comparison between the two identical boundary value problems - the contact between the two identical deformable spheres (Fig. 2(a)) and the contact between a deformable sphere and a rigid half-space (Fig. 2(d)) - shows that this cannot be true, since the ratio $a / R_{0}$ has different values in the two cases for the same value of contact radius $a$. Thus, it appears that a general transition criterion for the onset of the finite deformation regime does not exist. Nonetheless, throughout the article we shall present the results using the similarity scaling (1.3).

\section{Contact between deformable and rigid solids}

Consider indentation of a deformable sphere of radius $R_{\mathrm{d}}$ and a rigid sphere of radius $R_{\mathrm{r}}$. The following cases are considered:

(i) $R_{\mathrm{d}} / R_{\mathrm{r}}=0$, a deformable sphere indented by a rigid half-space (Fig. 2(d)),

(ii) $R_{\mathrm{d}} / R_{\mathrm{r}}=1$, a deformable sphere indented by a rigid sphere of the same size (Fig. 2(b)), and

(iii) $R_{\mathrm{d}} / R_{\mathrm{r}}=\infty$, a rigid sphere indenting a deformable half-space (Fig. 2(c)). Selected results are taken from (I) for this case.

Indentation results are presented for these three geometries, first for the case of an elastic-ideally plastic solid, and then for the case of an elastic-strain hardening solid. 


\subsection{Elastic-ideally plastic solids}

The indentation response of a rigid sphere against an elastic-ideally plastic sphere is presented in Fig. 4. The role of yield strain and the relative size of spheres is explored, and the results are presented in the form of a plot of average contact pressure versus contact size (Fig. 4(a) and (b)), contact area versus indent depth (Fig. 4(c) and (d)) and contact force versus indent depth (Fig. 4(e)).

First consider the finite element predictions of the average indentation pressure plotted against $\left(a E^{*} / R_{0} \sigma_{0}\right)$ in Fig. 4(a), for a range of values of $E^{*} / \sigma_{0}$, and for the geometries (i) - (iii) listed above. Here, $\sigma_{0}=\sigma_{1}$ equals the yield strength of the deformable solid. The initial stage of the elastic Hertzian indentation exists for $\left(a E^{*} / R_{0} \sigma_{0}\right)$ less than 2.5. This regime is not considered further here. For $\left(a E^{*} / R_{0} \sigma_{0}\right)$ less than about 50, the average pressure increases with $\left(a E^{*} / R_{0} \sigma_{0}\right)$; Johnson $(1970,1985)$ refers to this as the elastic-plastic regime. At higher values of $\left(a E^{*} / R_{0} \sigma_{0}\right)$, a plateau value of pressure is attained for all geometries in agreement with the value predicted by the rigid - ideally plastic similarity solution (1.4). However, we note that for sufficiently high values of yield strain, the similarity solution is not attained and the peak pressure is lower than the value predicted by the similarity solution.

At somewhat larger values of $\left(a E^{*} / R_{0} \sigma_{0}\right)$, the average pressure falls with increasing contact size and the curves markedly diverge. We shall refer to this regime as the finite deformation regime. At these large values of contact size, the elastic contribution to the strain field beneath the indenter is negligible, and the parameter $\left(a E^{*} / R_{0} \sigma_{0}\right)$ ceases to uniquely define the degree of indentation. For this regime, a plot of normalised contact pressure versus $a / R_{0}$ in Fig. 4 (b) indicates that $a / R_{0}$ characterises the contact pressure for a given geometry, but does not bring together the data for different geometries. Within the finite deformation regime, the average pressure diminishes fast with increasing $a / R_{0}$ for the case (i) of a deformable sphere between rigid platens, $R_{\mathrm{d}} / R_{\mathrm{r}}=0$, and slowest for the case (iii) of Brinell indentation, $R_{\mathrm{d}} / R_{\mathrm{r}}=\infty$. The drop in the average pressure with increasing contact size for $a / R_{0}>0.16$ for the case of Brinell indentation represents a failure of the assumptions involved in the similarity solution, specifically the assumption of infinitesimal strain kinematics and the boundary condition of uniform normal velocity. As the contact size increases, the tangential velocity of points in contact with the indenter deviates from the horizontal, so that the uniform vertical velocity boundary condition ceases to be appropriate. For geometries (i) and (ii), the deformable body is of finite size. As the contact size increases, the assumption that the body can be treated as a semi-infinite solid (assumption (2) of section 1.1) ceases to be appropriate. Thus, the drop in the average pressure occurs at a lower value of $a / R_{0}$ for cases (i) and (ii) than for case (iii). This large drop in the average pressure for large contacts has been observed experimentally for the compression of elastic-plastic spheres between flat elastic platens (Chaudhri et al., 1984; Timothy et al., 1987; Chaudhri, 1987).

The normalised contact area $\left(a^{2} / 2 h R_{0}\right)$ is plotted against $\left(a E^{*} / R_{0} \sigma_{0}\right)$ in Fig. $4(\mathrm{c})$ and against $a / R_{0}$ in Fig. 4(d). As noted above in the discussion of contact pressure, it is clear that the non-dimensional group $\left(a E^{*} / R_{0} \sigma_{0}\right)$ is the governing parameter for the elastic-plastic regime. With a further increase in contact size, the rigid-plastic similarity regime of constant $\left(a^{2} / 2 h R_{0}\right)$ is attained only for very low values of the yield strain. Finally, for large $a / R_{0}$ values, the normalised contact area $\left(a^{2} / 2 h R_{0}\right)$ decreases with increasing $a / R_{0}$. Within this regime, the contact area depends upon both the type of geometry and, for high values of yield strain, the magnitude of the yield strain.

The relation between the indent depth and the contact force is of fundamental importance in applications of indentation theory to the contact stiffness of rough surfaces and to the macroscopic compaction response of an aggregate of particles. Plots of the normalised contact load $\left(F / \pi R_{0}^{2} \sigma_{0}\right)$ versus the normalised indent depth $h / R_{0}$ are given in Fig. 4(e) for the same range of values of yield strain as reported in Fig. 4(a-d). The range of indentation depths of interest in powder compaction falls within the finite deformation regime. The contact stiffness is lower than predicted by the similarity solution, and this is due to the multiplicative effect of the lower contact pressure (Fig. 4(b)) and the smaller contact area (see Fig. 4(d)), within the finite deformation regime. 

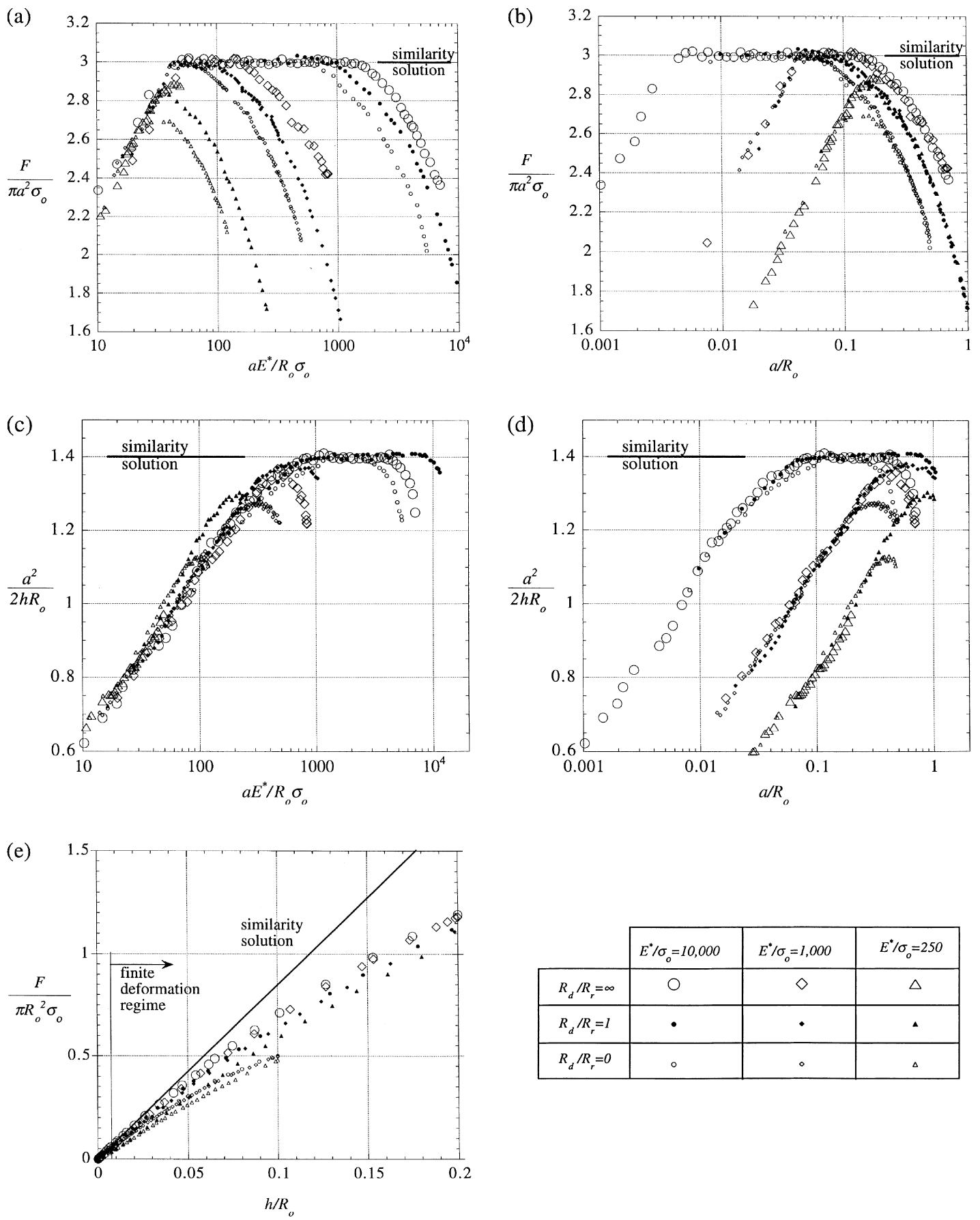

\begin{tabular}{|c|c|c|c|}
\cline { 2 - 4 } \multicolumn{1}{c|}{} & $E^{*} / \sigma_{o}=10,000$ & $E^{*} / \sigma_{o}=1,000$ & $E^{*} / \sigma_{o}=250$ \\
\hline$R_{d} / R_{r}=\infty$ & 0 & $\diamond$ & $\Delta$ \\
\hline$R_{d} / R_{r}=1$ & $\bullet$ & $\bullet$ & $\Delta$ \\
\hline$R_{d} / R_{r}=0$ & $\circ$ & $\diamond$ & $\Delta$ \\
\hline
\end{tabular}

Fig. 4. Comparison of the similarity solution and finite element results for the elastic-ideally plastic solid: (a) average pressure as a function of $a E^{*} / R_{0} \sigma_{0}$, (b) average pressure as a function of $a / R_{0}$, (c) $a^{2} / 2 h R_{0}$ as a function of $a E^{*} / R_{0} \sigma_{0}$, (d) $a^{2} / 2 h R_{0}$ as a function of $a / R_{0}$, (e) Load-displacement curves. In each figure, results are shown for (i) a sphere indented by a rigid half-space: small open symbols; (ii) a sphere indented by a rigid sphere of equal size: solid symbols; and (iii) a half-space indented by a rigid sphere: large open symbols. Circles: $E^{*} / \sigma_{0}=10,000$, Diamonds: $E^{*} / \sigma_{0}=1000$, Triangles: $E^{*} / \sigma_{0}=250$. 


\subsection{Elastic-hardening solids}

Practical engineering alloys strain harden in the plastic range, and it is instructive to explore the effects of strain hardening on the indentation response for an idealised elastic-strain hardening solid as characterised by Eq. (2.1). Here, we report only the results for a solid which strain hardens strongly such as annealed copper, with $m=3$. Additional calculations were performed for $m=5$ and the results follow the same general trends as discussed here for $m=3$. On noting that the similarity solution (1.4) states that the average indentation pressure scales with the reference stress

$$
\sigma_{r}=\sigma_{\mathrm{e}}\left(\frac{a}{R_{0}}\right)^{1 / m},
$$

we present results for the average indentation pressure by normalising stresses with respect to $\sigma_{r}$. For selected values of yield strain and for $m=3$, predictions for the reduced average pressure $\left(F / \pi a^{2} \sigma_{r}\right)$ are given in Fig. 5(a) and (b) and for the normalised contact area $a^{2} / 2 h R_{0}$ in Fig. 5(c) and (d). The same three geometries (i)-(iii) are considered as defined at the start of Section 3 and as reported for the elastic-ideally plastic solid in Fig. 4(a-e).

For all three geometries and for all values of yield strain considered, the average contact pressure $\left(F / \pi a^{2} \sigma_{r}\right)$ increases with increasing $\left(a E^{*} / R_{0} \sigma_{0}\right)$ until $\left(a E^{*} / R_{0} \sigma_{0}\right)$ is about 100 , (see Fig. 5(a)). At higher values of $\left(a E^{*} / R_{0} \sigma_{0}\right)$, the similarity regime is attained provided the yield strain is sufficiently small: $\sigma_{0} / E^{*}$ less than about 0.0003 , as shown in (I). The finite deformation regime is entered when $a / R_{0}$ exceeds approximately 0.06 for case (i) and 0.1 for case (ii), as shown in Fig. 5(b). No drop in the reduced average pressure is observed at large contact sizes for Brinell indentation, case (iii), but if the deformable body is of finite size, cases (i) and (ii), the reduced average pressure drops with increasing contact size.

The evolution of the normalised contact area with contact size (Fig. 5(c) and (d)), for the strain hardening case, $m=3$ is qualitatively the same as in the elastic-ideally plastic case (Fig. 4(c) and (d)). To within numerical error, the similarity solution is attained only for very low values of yield strain. The parameter $a^{2} / 2 h R_{0}$ increases with increasing $\left(a E^{*} / R_{0} \sigma_{0}\right)$ in the elastic-plastic regime, remains constant in the fully plastic similarity regime and then decreases again in the finite deformation regime.

The similarity solution (1.6) predicts that for $m=3$, the normalised indentation load $\left(F / \pi R_{0}^{2} \sigma_{\mathrm{e}}\right)$ is proportional to the normalised indent depth, $h / R_{0}$ to the power of $7 / 6$. Numerical results show that this contact law breaks down within the finite deformation regime (Fig. 5(e)), but the deviation from the similarity solution is less pronounced than in the elastic-ideally plastic case (Fig. 4(e)). The effect of elasticity upon contact stiffness is stronger than the effect of the relative diameter of spheres.

\section{Contact between two deformable solids}

In this section, we discuss the frictionless normal indentation between two deformable spheres with radii $R_{1}$ and $R_{2}$. In particular, we explore the effect of relative size $R_{1} / R_{2}$, for two spheres made of the same material. We consider two extreme cases:

Case ( $A$ ): Two identical spheres with $R_{1}=R_{2}$, as shown in Fig. 2(a), and

Case (B): A sphere of radius $R_{1}$ indenting a half-space (radius $R_{2}=\infty$ ), as shown in Fig. 2(e).

Case (A), the indentation of two identical spheres, is an identical boundary value problem to that of a deformable sphere pressed against a rigid half-space (Fig. 2(d)), discussed as case (i) in Section 3. The 

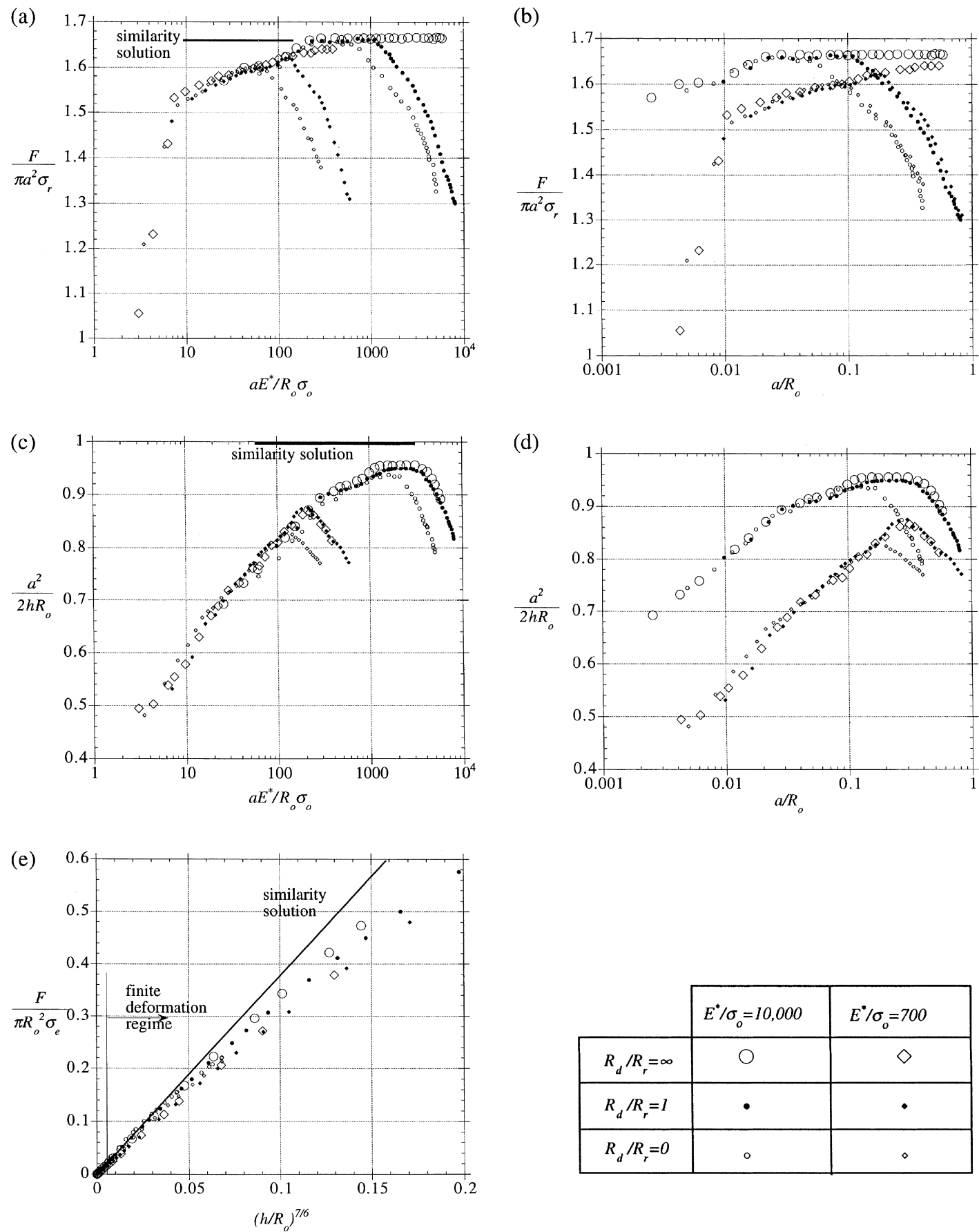

\begin{tabular}{|c|c|c|}
\cline { 2 - 3 } \multicolumn{1}{c|}{} & $E^{*} / \sigma_{o}=10,000$ & $E^{*} / \sigma_{o}=700$ \\
\hline$R_{d} / R_{r}=\infty$ & $\bigcirc$ & $\diamond$ \\
\hline$R_{d} / R_{r}=1$ & $\bullet$ & $\bullet$ \\
\hline$R_{d} / R_{r}=0$ & $\circ$ & $\diamond$ \\
\hline
\end{tabular}

Fig. 5. Comparison of the similarity solution and finite element results for the elastic-power-law hardening solid, with $m=3$ : (a) reduced average pressure, $F / \pi a^{2} \sigma_{r}$, as a function of $a E^{*} / R_{0} \sigma_{0}$, (b) reduced average pressure as a function of $a / R_{0}$, (c) $a^{2} / 2 h R_{0}$ as a function of $a E^{*} / R_{0} \sigma_{0}$, (d) $a^{2} / 2 h R_{0}$ as a function of $a / R_{0}$, (e) load-displacement curve. In each figure results are shown for (i) a sphere indented by a rigid half-space: small open symbols; (ii) a sphere indented by a rigid sphere of equal size: solid symbols; and (iii) a halfspace indented by a rigid sphere: large open symbols. Circles: $E^{*} / \sigma_{0}=10,000$, Diamonds: $E^{*} / \sigma_{0}=700$. 
results for two identical spheres are easily obtained by rescaling the results for the case of a deformable sphere pressed against a rigid half-space, as follows. In both problems, $h$ is defined as the total overlap of the bodies in contact (see Fig. 1). The definitions of the equivalent radius $R_{0}$ and the equivalent strength $\sigma_{\mathrm{e}}$ imply the following relations. On denoting the case of two identical sphere by a superscript (A) and the case of a deformable sphere pressed against a rigid half-space by a superscript $(i)$, we have for a given contact radius $a$,

$$
R_{0}^{(\mathrm{A})}=(1 / 2) R_{0}^{(i)}, \sigma_{\mathrm{e}}^{(\mathrm{A})}=2^{-1 / m} \sigma_{\mathrm{e}}^{(i)} \quad \text { and } \quad h^{(\mathrm{A})}=2 h^{(i)} .
$$

Now, consider case (B). Initially, the sphere and the half-space both deform and contribute to the total overlap $h$ as suggested by the similarity solution. As the contact size increases, the deformation is concentrated within the sphere. Deformed shapes for the case (B) are shown in Fig. 6(a) and (b) for the cases $m=3$ and $m=\infty$. When both the solids are elastic-ideally plastic (Fig. 6(a)), the half-space is practically rigid. In the elastic-hardening case, the contribution of the half-space deformation to the overall displacement is minor. This can be explained as follows: At large $a / R_{0}$ values, the average contact pressure for a deformable sphere on a rigid half-space is lower than the average contact pressure for a rigid sphere indenting a deformable half-space (see Fig. 4(b)). Thus, the sphere undergoes larger plastic deformation than the half-space for the same (large) value of $a / R_{0}$.

The force-displacement plots for the contact of deformable spheres, cases (A) and (B), are shown in Fig. 7(a) for elastic-ideally plastic solids and in Fig. 7(b) for elastic-hardening solids with $m=3$. For both values of $m$, the contact stiffness is weakly dependent upon the relative size of the spheres for $h / R_{0}<0.1$. At larger values of $h / R_{0}$, the contact force depends more strongly upon the relative size of spheres, and the curves diverge.

\section{Indentation maps}

It is instructive to construct indentation maps, first for the contact between a deformable and a rigid sphere (Fig. 8(a)), and second, for two deformable spheres (Fig. 8(b)). The appropriate axes are $a / R_{0}$ and $\sigma_{0} / E^{*}$, where $R_{0}$ is defined in Eq. (1.3) and $\sigma_{0}$ is the lower of the two initial yield strengths:
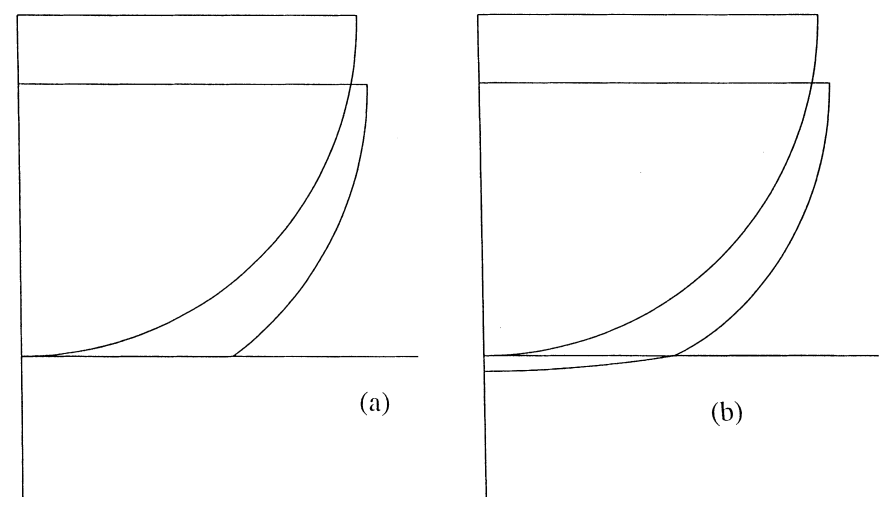

Fig. 6. Initial and deformed configuration for the contact of a deformable sphere and a deformable half-space, case (B): (a) elasticideally plastic solids, $E^{*} / \sigma_{0}=5000, a / R_{0}=0.6$; (b) elastic-hardening solids, $m=3, E^{*} / \sigma_{0}=5000, a / R_{0}=0.5$. 

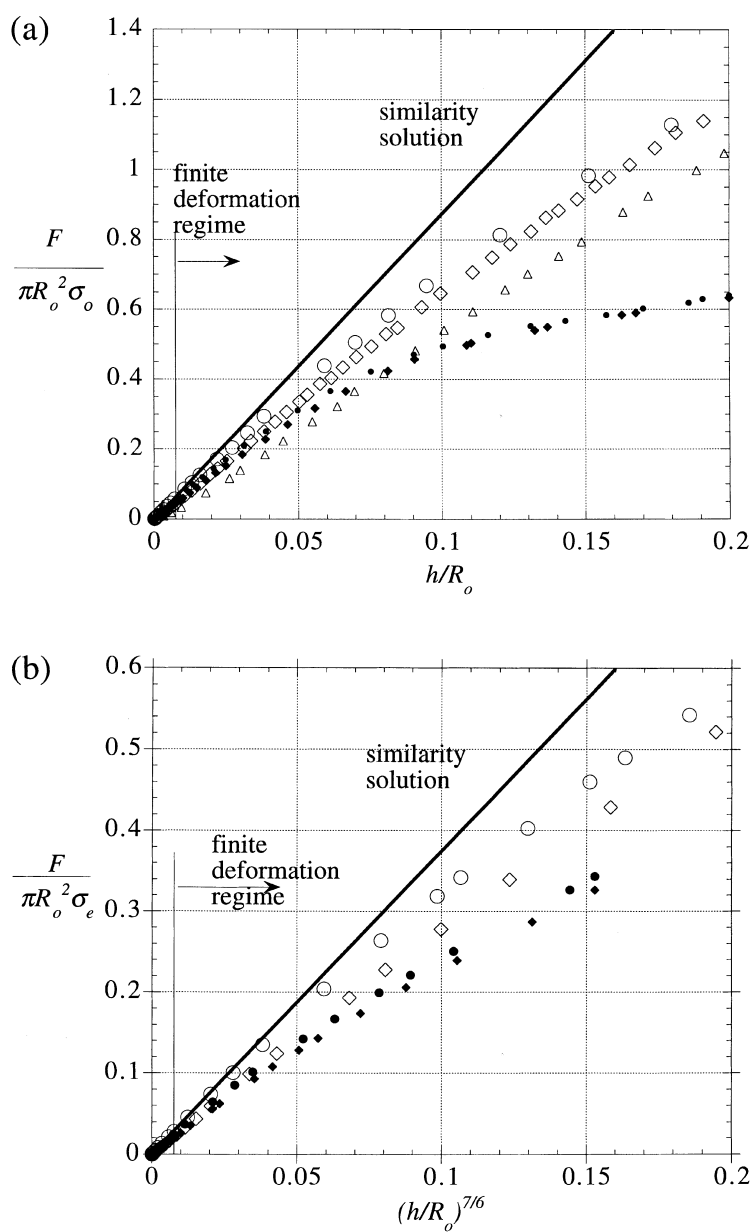

Fig. 7. Load-displacement curves for the indentation of two identical deformable spheres, case (A), shown by open symbols, and indentation of a deformable sphere and a deformable half-space, case (B), shown by solid symbols: (a) elastic-ideally plastic solids, Circles: $E^{*} / \sigma_{0}=5000$, Diamonds: $E^{*} / \sigma_{0}=500$, Triangles: $E^{*} / \sigma_{0}=125$; (b) elastic-hardening solids, $m=3$. Circles: $E^{*} / \sigma_{0}=5000$, Diamonds: $E^{*} / \sigma_{0}=350$.

$$
\sigma_{0}=\min \left\{\sigma_{01}, \sigma_{02}\right\}
$$

where $\sigma_{01}$ and $\sigma_{02}$ are defined in Eq. (2.1b).

A detailed indentation map has already been developed for the case of Brinell indentation in (I). Here, we build on the results of (I) and develop indentation maps for the contact between a deformable sphere with radius $R_{\mathrm{d}}$ and a rigid sphere with radius $R_{\mathrm{r}}$, for relative sizes $R_{\mathrm{d}} / R_{\mathrm{r}}=0,1$ and $\infty$, and for $m=3$ and $\infty$ (Fig. 8(a)). We define a fully plastic, similarity regime as a region of $a / R_{0}-\sigma_{0} / E^{*}$ space in which both the reduced average pressure $F / \pi R_{0}^{2} \sigma_{\mathrm{e}}$ and the normalised contact size $a^{2} / 2 h R_{0}$ equal the values given by the similarity solution, and are independent of the contact size. The finite deformation regime is defined as a region of $a / R_{0}-\sigma_{0} / E^{*}$ space in which either the reduced average pressure $F / \pi R_{0}^{2} \sigma_{\mathrm{r}}$ or the normalised contact size $a^{2} / 2 h R_{0}$ decrease with increasing contact size. For a given value of strain hardening exponent $m$, the boundaries between the elastic and elastic-plastic regimes, and between the elastic-plastic and 

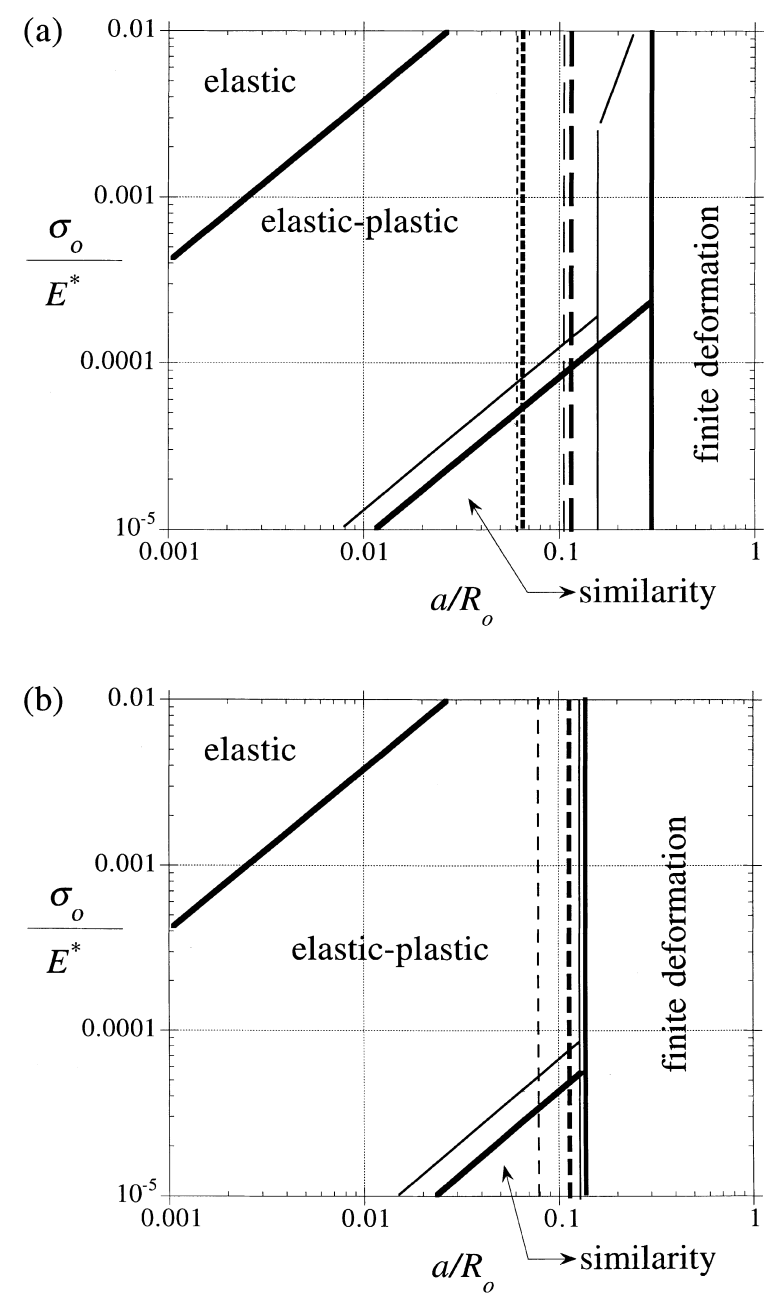

Fig. 8. (a) Indentation maps for the contact between a rigid sphere and a deformable sphere: The deformable sphere is elastic-ideally plastic (thin lines), and elastic-power-law hardening with $m=3$ (thick lines). The ratio between the radius of the deformable sphere $R_{\mathrm{d}}$ and the radius of the rigid sphere $R_{\mathrm{r}}$ is given by (i) $R_{\mathrm{d}} / R_{\mathrm{r}}=0$, short dashed lines; (ii) $R_{\mathrm{d}} / R_{\mathrm{r}}=1$, long dashed lines; and (iii) $R_{\mathrm{d}} / R_{\mathrm{r}}=\infty$, full lines; (b) Indentation maps for the contact between two deformable spheres: The solid is elastic-ideally plastic (thin lines), and elastic-power-law hardening with $m=3$ (thick lines). The ratio between the radii of deformable spheres $R_{1}$ and $R_{2}$ is: $R_{1} / R_{2}=1$ for case (A), full lines; $R_{1} / R_{2}=0$ for case (B), dashed lines.

similarity regimes are identical for all three geometries, as expected from the Hertz solution and the similarity solution. The onset of the finite deformation regime is governed by the relative size of the deformable sphere: the smaller the relative size of the deformable sphere, the smaller is the critical contact radius $a / R_{0}$ for the onset of the finite deformation regime. Strain hardening has a significant effect on the similarity/ finite deformation boundary only for the case of the rigid sphere and deformable half-space; as $m$ decreases from $\infty$ to 3 , the onset of the finite deformation regime shifts to a larger value of $a / R_{0}$ by a factor of about 2 .

Indentation maps for the contact between two identical deformable spheres (case A), and between a deformable sphere and a deformable half-space (case B), are shown in Fig. 8(b), for $m=3$ and $m=\infty$. 
The similarity regime is very difficult to achieve for the case of contact between two deformable bodies. An extremely low value of $\sigma_{0} / E^{*}$ is needed which makes the associated computations expensive. None of the cases shown in Fig. 7 falls within the similarity regime. Boundaries of the similarity regime in Fig. 8(b) are estimated by extrapolation of the results reported in Section 4, using the following arguments:

(i) For a given hardening exponent $m$, the boundary between the elastic-plastic and similarity regimes is a function of $\left(a E^{*} / R_{0} \sigma_{0}\right)$ only.

(ii) For a given $m$ and a given relative size of particles, the boundary between the similarity regime and the finite deformation regime depends only upon $a / R_{0}$.

The elastic/elastic-plastic and elastic-plastic/similarity boundaries are again independent of the relative size of the spheres. The similarity/finite deformation boundaries for the two geometries almost coincide for $m=3$ and differ by a factor of about 2 in $a / R_{0}$ value for $m=\infty$. This can be traced back to the feature that, for $m=\infty$, the half-space deforms much less than the sphere, as discussed in Section 4.

\section{Compaction of a 3-D unit cell}

Micromechanical constitutive models for the stage I compaction of powders (Fleck et al., 1992; Fleck 1995; Storakers et al., 1999) rely upon the assumption that there is no cross-coupling between the forcedisplacement laws for each contact of a particle: it is assumed that each contact behaves independently. A three-dimensional calculation of the response of a random assembly of particles is prohibitively expensive. Here, we estimate the lowest relative density for which contacts cease to behave independently. For that purpose, we seek to define the periodic arrangement which is most likely to promote the contact interaction. Based on the present experience and previous work (Ogbonna and Fleck, 1995), one concludes that the contact interaction is promoted by

1. isostatic compaction (rather than closed die compaction),

2. contacts between soft and rigid particles,

3. a strong hardening response, and

4. contact between spheres of equal size.

Thus, we select an ordered binary mix of deformable and rigid spheres, of equal size and equal volume fraction. The B2 unit cell, based on two overlapping simple cubic lattices is shown in Fig. 2(g). Each deformable sphere has eight rigid nearest neighbours and six deformable next nearest neighbours. This arrangement is chosen because its initial density, $D_{0}=0.68$ is close to the dense random packing density of 0.64 , and because the 50-50 mix of metal ceramic spheres gives a highly symmetric configuration. By considering isostatic compaction, the symmetry can be exploited to minimise the number of degrees of freedom in the finite element model. The mesh in Fig. 3(b) models a 1/24 of the central soft sphere. A rigid sphere initially in contact with the central sphere is modelled as a spherical rigid surface. During the course of compaction, the number of contacts is allowed to increase from 8 to 14; the additional six contacts are with deformable next nearest neighbours and are modelled as contacts with flat rigid surfaces.

The deformable sphere is taken to be elastic-plastic with a hardening index $m=3$ and $E^{*} / \sigma_{0}=700$. These values are chosen to maximise the convergence rate. Since the analysis takes about 30 days of CPU time on a fast workstation, these are important considerations. With this choice of yield strain, the similarity regime is not expected (see Fig. 4). Nevertheless, the 3-D simulation of isostatic compaction provides a useful assessment of the accuracy of the simpler axisymmetric models. We compare the following cases: 

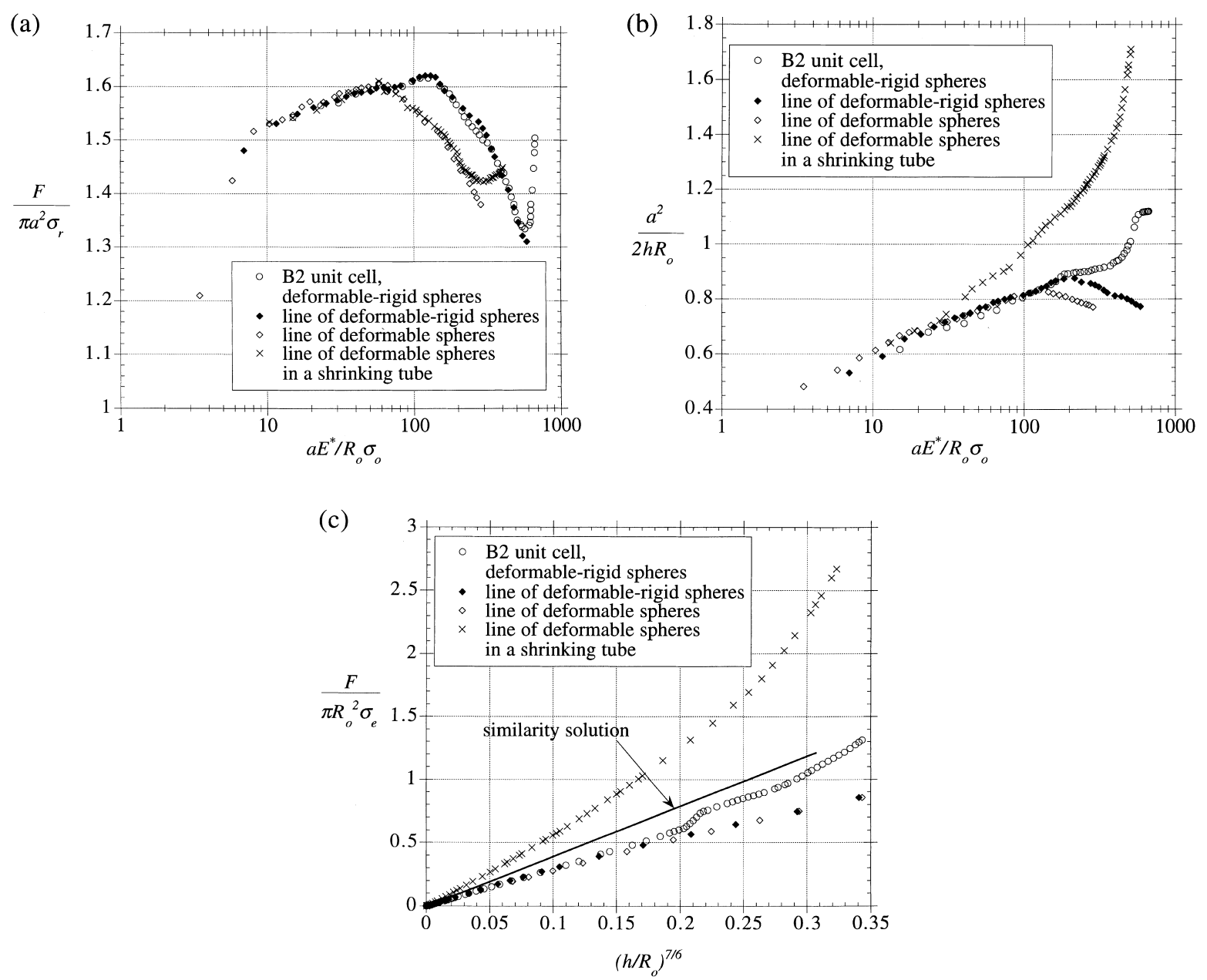

Fig. 9. Effect of contact-contact interaction on the contact force. Comparison between a line of identical deformable spheres, a line of deformable and rigid spheres of equal diameter, a shrinking-tube model of isostatic compaction and three-dimensional isostatic compaction of a periodic B2 unit cell: (a) reduced average pressure, $F / \pi a^{2} \sigma_{r}$ as a function of $a E^{*} / R_{0} \sigma_{0}$, (b) $a^{2} / 2 h R_{0}$ as a function of $a E^{*} / R_{0} \sigma_{0}$, (c) Load-displacement response.

I. uniaxial compaction of a line of identical deformable spheres (Fig. 2(a)) and a line of alternating deformable and rigid spheres of equal diameter (Fig. 2(b)),

II. an axisymmetric model of isostatic compaction (Fig. 2(f)), in which the shrinking cylinder enforces a radial strain rate equal to the axial strain rate, and

III. the three-dimensional isostatic compaction of a periodic B2 cell (Fig. 2(g)).

The evolution of the reduced average pressure with the non-dimensional variable $a E^{*} / R_{0} \sigma_{0}$ is shown in Fig. 9(a) for cases I-III. In the elastic-plastic regime, $F / \pi a^{2} \sigma_{r}$ increases with $a E^{*} / R_{0} \sigma_{0}$, and all the curves coincide. At the start of the finite deformation regime, the response is again independent of the type of loading, but the average pressure is greater for the contacts between deformable and rigid spheres than for the contacts between deformable spheres. The contact pressure depends upon the loading arrangement only in the latter parts of the finite deformation regime. 
Next, consider the normalised contact area $a^{2} / 2 h R_{0}$, plotted against $a E^{*} / R_{0} \sigma_{0}$ in Fig. 9 . The contact area becomes sensitive to the lateral constraint at a lower value of $a E^{*} / R_{0} \sigma_{0}$ than the contact pressure $F / \pi a^{2} \sigma_{r}$. The axisymmetric model for isostatic compaction (case II) is over-constrained and predicts much greater contact-contact interaction than the more realistic B2 unit cell.

The force-displacement relations are plotted in Fig. 9(c), with axes $\left(h / R_{0}\right)^{7 / 6}$ and $F / \pi R_{0}^{2} \sigma_{\mathrm{e}}$. It is clear that the axisymmetric model for isostatic compaction (case II) predicts too stiff a response. Ogbonna and Fleck (1995) have also compared the compaction response of a line of deformable spheres (case I) with a line of deformable spheres constrained by a shrinking tube (case II). They too find that the highly constrained axisymmetric model gives a much stiffer response than the unconstrained line of deformable spheres (see Fig. 10 of their article). The more realistic results for the B2 unit cell (case III) indicate that the contacts behave independently up to $\left(h / R_{0}\right)^{7 / 6}=0.2$, which corresponds to the relative density $D=0.826$. The sudden increase in the contact force of the B2 unit cell for $\left(h / R_{0}\right)^{7 / 6}$ between 0.20 and 0.22 is due to the simultaneous creation of six new contacts.

With the exception of case II (the over-constrained line of deformable spheres), the contact force versus indent depth response is almost identical for all loading cases, for $\left(h / R_{0}\right)^{7 / 6}$ in the range $0-0.2$. For these cases, the contact load is somewhat below than that given by the similarity solution; this is expected, as the relevant results are within the finite deformation regime, and the assumed value of the yield strain is large.

\section{Concluding remarks}

We have constructed indentation maps for frictionless normal indentation of deformable spheres, and for the indentation of a deformable sphere by a rigid sphere. The axes of the map are the normalised contact radius $a / R_{0}$ and a measure of the initial yield strain, $\sigma_{0} / E^{*}$ (see Eqs. (1.3), (2.4) and (5.1)). The general features of both maps can be summarised as follows. First, the boundary between the elastic and the elastic-plastic regimes is independent of both the relative size of the spheres and the strain hardening exponent. Second, the boundary between the elastic-plastic and the similarity regimes depends somewhat on the level of strain hardening but is independent of the relative size of the spheres. And third, the position of the finite deformation regime depends both on the degree of strain hardening and the relative size of the spheres.

For the purpose of building a constitutive model for cold powder compaction, we require the force versus displacement contact law between dissimilar spheres for indentation depths in the range $h / R_{0}=0-0.2$. The contact stiffness is reasonably well approximated by the similarity power law (1.6) but with a modified value for the stiffness parameter $K$, as defined in Eq. (1.6b). We define $K_{\mathrm{FEM}}$ as the value for $K$ at $h / R_{0}=0.1$ from the numerical simulations solution. $K_{\text {theory }}$ is the value for $K$ given by the similarity solution, (see Eq. (1.6b). The ratio $K_{\mathrm{FEM}} / K_{\text {theory }}$ lies in the narrow range of $0.55-0.87$ for all geometries considered and for the strain hardening exponent in the range $m=3$ to $m=\infty$. Consistently, $K_{\mathrm{FEM}} / K_{\text {theory }}$ increases with decreasing $m$ (see Fig. 10). The contact between two deformable spheres gives slightly lower values for $K_{\mathrm{FEM}} / K_{\text {theory }}$ than the contact between a deformable and a rigid sphere, for a given relative size and strain hardening exponent.

We have shown that the assumption of independent contacts holds to about $D=0.82$, for the case of isostatic compaction of a $\mathrm{B} 2$ unit cell of deformable and rigid spheres, with $m=3$. It is reasonable to assume that for higher values of $m$ and for the isostatic compaction of identical deformable spheres, the assumption of independent contacts will hold to higher relative densities than $D=0.82$. We conclude that the assumption of independent deformation at contacts is reasonable for stage I powder compaction. 


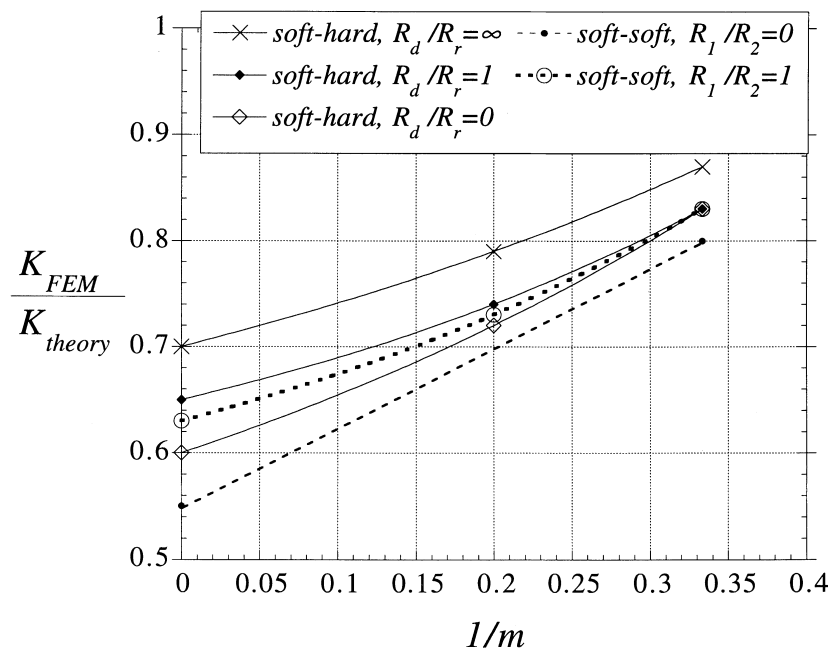

Fig. 10. The ratio of the finite element stiffness parameter $K_{\mathrm{FEM}}$ and the similarity stiffness parameter $K_{\text {theory }}$ as defined in Eq. (1.6b). $K_{\text {FEM }}$ is obtained from Eq. (1.6a) using the values of force at $h / R_{0}=0.1$ for the cases with the lowest yield strain.

\section{Acknowledgements}

The authors are grateful for the financial support from NIST, contract number 70-NANB5H0042, and for the helpful technical discussions with Dr. R.J. Fields of NIST. The authors also acknowledge the helpful comments by Prof. K.L. Johnson and Dr. M.M. Chaudhri.

\section{References}

ABAQUS, 1995. V5.5 User's Manual and Theory Manual. Hibbitt, Karlsson and Sorensen.

Biwa, S., Storakers, B., 1995. An analysis of fully plastic Brinell indentation. J. Mech. Phys. Solids 43, $1303-1334$.

Bower, A.F., Fleck, N.A., Needleman, A., Ogbonna, N., 1993. Indentation of power law creeping solids. Proc. Royal Soc. London A441, 97-124.

Chaudhri, M.M., 1987. The plastic deformation of single asperities by hard flats. Inst. Mech. Engng. Conference Publications C158, 1003-1012.

Chaudhri, M.M., Hutchings, I.M., Makin, P.L., 1984. Plastic compression of spheres. Phil. Mag. A 49 (4), $493-503$.

Fleck, N.A., 1995. On the cold compaction of powders. J. Mech. Phys. Solids 43, 1409-1431.

Fleck, N.A., Kuhn, L.T., McMeeking, R.M., 1992. Yielding of metal powder bonded by isolated contacts. J. Mech. Phys. Solids 40, 1139-1162.

Gampala, R., Elzey, D.M., Wadley, H.N.G., 1994. Plastic deformation of asperities during consolidation of plasma sprayed metal matrix composite monotape. Acta Metall. 42, 3209-3221.

Hill, R., 1992. Similarity analysis of creep indentation tests. Proc. Roy. Soc. London A436, 617-630.

Hill, R., Storakers, B., Zdunek, A.B., 1989. A theoretical study of the Brinell hardness test. Proc. Royal Soc. London A423, 301-330.

Johnson, K.L., 1970. The correlation of indentation experiments. J. Mech. Phys. Solids 18, 115-126.

Johnson, K.L., 1985. Contact Mechanics. Cambridge University Press. London.

Mesarovic, S.Dj., Fleck, N.A., 1999. Spherical Indentation of elastic-plastic solids, Proc. Roy. Soc. Lond. A455, $2707-2728$.

Ogbonna, N., Fleck, N.A., 1995. Compaction of an array of spherical particles. Acta Metall. 43, 603-620.

Sternberg, E., Rosenthal, F., 1952. The elastic sphere under concentrated load. J. Appl. Mechanics 19, 413-421.

Storakers, B., 1997. Local contact behaviour of viscoplastic particles. In: Fleck, N.A., Cocks, A.C.F., (Eds.) Proc. IUTAM Symp. on Mechanics of Granular Flow and Powder Compaction. Kluwer Academic Publishers. Dordrecht. July 1996. 
Storakers, B., Biwa, S., Larsson, P.-L., 1997. Similarity analysis of inelastic contact. Int. J. Solids Struct. 34 (24), $3061-3083$.

Storakers, B., Fleck, N.A., McMeeking, R.M., 1999. The viscoplastic compaction of composite powders, J. Mech. Phys. Solids, 47, 785-815.

Timothy, S.P., Pearson, J.M., Hutchings, I.M., 1987. The contact pressure distribution during plastic compression of lead spheres. Int. J. Mech. Sci. 79, 713-719. 